Annales Geophysicae (2002) 20: 817-822 (c) European Geophysical Society 2002

\title{
The bottomside parameters $B 0, B 1$ obtained from incoherent scatter measurements during a solar maximum and their comparisons with the IRI-2001 model
}

\author{
N. K. Sethi and K. K. Mahajan \\ Radio and Atmospheric Sciences Division, National Physical Laboratory, Dr. K. S. Krishnan Road, New Delhi - 110012 , \\ India
}

Received: 6 September 2001 - Revised: 23 January 2002 - Accepted: 12 February 2002

\begin{abstract}
High resolution electron density profiles $(\mathrm{Ne})$ measured with the Arecibo (18.4 N, 66.7 W), Incoherent Scatter radar (I.S.) are used to obtain the bottomside shape parameters $B 0, B 1$ for a solar maximum period (198990). Median values of these parameters are compared with those obtained from the IRI-2001 model. It is observed that during summer, the IRI values agree fairly well with the Arecibo values, though the numbers are somewhat larger during the daytime. Discrepancies occur during winter and equinox, when the IRI underestimates $B 0$ for the local times from about 12:00 LT to about 20:00 LT. Furthermore, the IRI model tends to generally overestimate $B 1$ at all local times. At Arecibo, $B 0$ increases by about $50 \%$, and $B 1$ decreases by about $30 \%$ from solar minimum to solar maximum.
\end{abstract}

Key words. Ionosphere (equational ionosphere; modeling and forecasting)

\section{Introduction}

The International Reference Ionosphere (IRI) is a global empirical model which specifies the monthly average of the electron density, electron temperature, ion temperature and ion composition. Over the years, testing and modification of IRI has continued with extensive participation by a joint working group of the International Union of Radio Science (URSI) and the Committee on Space Research (COSPAR). These efforts have led to improvements through several versions (IRI-78, IRI-80, IRI-86, IRI-90, IRI-95, IRI-2001).

Electron density distribution in the IRI model below the F2 peak is described by the analytic function (Ramakrishnan and Rawer, 1972):

$N(h)=N m \mathrm{~F} 2 \times \exp \left(-x^{B 1}\right) / \cos h(x)$,

with $x=(h m \mathrm{~F} 2-h) / B 0$.

$B 0$ is the bottomside thickness parameter and $B 1$ determines the profile shape describing the bottomside electron

Correspondence to: N. K. Sethi (nksethi@csnpl.ren.nic.in) density distribution below the F2 peak. In the widely used IRI-90 (Bilitza, 1990), there were two options for providing $B 0$. The old option was the standard model which made use of a table of values of $B 0$ deduced from profile inversion of ionograms at mid-latitude stations. The second option (which was considered the better choice, especially at low-latitudes), used Gulyaeva's (1987) model for $B 0$. This was based on half density height $h 0.5$ (the height below the F2 peak, where the density fell off to half the peak value: $N(h 0.5)=0.5 \mathrm{Nm} \mathrm{F} 2)$. This $B 0$ was given as

$B 0=(h m \mathrm{~F} 2-h 0.5) / C$,

where $C$ was a function of $B 1$.

Since the IRI-90 assumed a constant value of 3 for $B 1$ most of the time, the parameter $C$ correspondingly was assigned a value of 0.75556 (Bilitza, 1990). In earlier studies, Mahajan et al. (1995), using Arecibo measurements (a lowlatitude station) during solar minimum period (1974-1977) showed that $B 0$ values based on Gulyaeva's 1987 model were significantly larger, especially during daytime, for summer and equinox seasons, than those obtained from the measurements. Later, Pandey and Sethi (1996), using I.S. measurements during daytime from Arecibo, for the solar minimum period (1974-1977), showed that the IRI-90 values of electron densities below the F2 peak at all the heights were larger during summer and equinox months. A comparison of $\mathrm{Ne}$ profiles derived from ionogram at low-latitudes during solar minimum and maximum periods, with those obtained from IRI-90, also showed similar results (De Gonzalez 1996; Aggarwal et al., 1996). Gulyaeva et al. (1996) compared the ratio $h 0.5 / \mathrm{Hm} \mathrm{F} 2$ using Arecibo $N e$ profiles during summer, with those obtained from the IRI-90 model. Their analysis showed that the IRI-90 underestimated the ratios at all local times in comparison to those obtained from the observations. In view of these and other discrepancies, the IRI Task Force in 1995 (Bilitza, 1996) decided to establish B0, B1 parameters directly from the measured profiles below the F2 peak down to $0.24 N m \mathrm{~F} 2$. Following this, efforts were made by several workers to establish a set of $B 0, B 1$ parameters, us- 

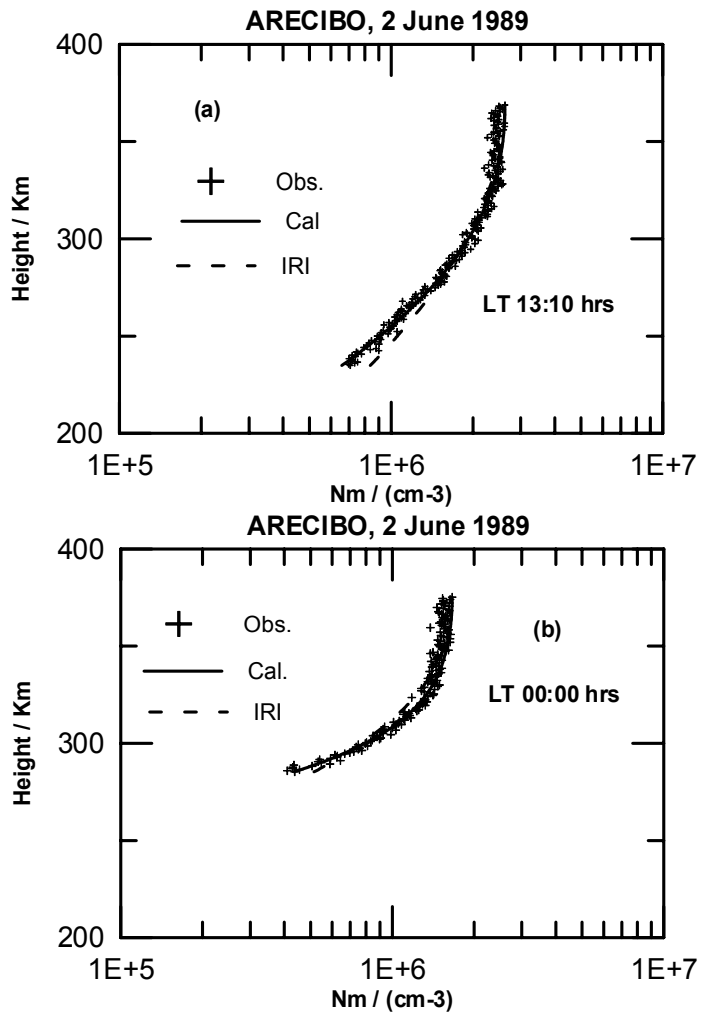

Fig. 1. Sample profiles obtained using best $B 0, B 1$ parameters derived from I.S. measurements at Arecibo for (a) daytime, (b) midnight. Calculated profiles are shown by solid lines. The IRI model profile is shown as dash line.

ing data from various stations (Huang and Reinisch, 1996; Adeniyi and Radicella 1998; Sethi et al., 2000; Mahajan and Sethi, 2001). Their results showed that $B 1$ varied between 1 and 9 with higher values by night and lower ones by day, while in the IRI-90, $B 1$ was kept at a constant value of 3 for most of the cases. These workers also reported that $B 0$ showed a strong diurnal variation with higher values around noon. In view of the discrepancies between the IRI-90 model and the ionosonde, as well as the radar measurements, the model has since been revised and named IRI-2001 (Bilitza, $2001)$. The $B 0, B 1$ values in the model are primarily based upon the ionosonde data base, generated through the ICTP efforts (Bilitza et al., 2000) and provide solar activity, as well as seasonal variations.

We have recently acquired incoherent scatter $N e-h$ data for the years 1989-1990 (a period of high solar activity) from the Arecibo observatory (Qihou Zhou, 2000, private communication). In this paper, we compute the $B 0, B 1$ parameters for these data and compare these values with those obtained during the solar minimum by Sethi et al. (2000), Mahajan and Sethi (2001), and with the IRI-2001.
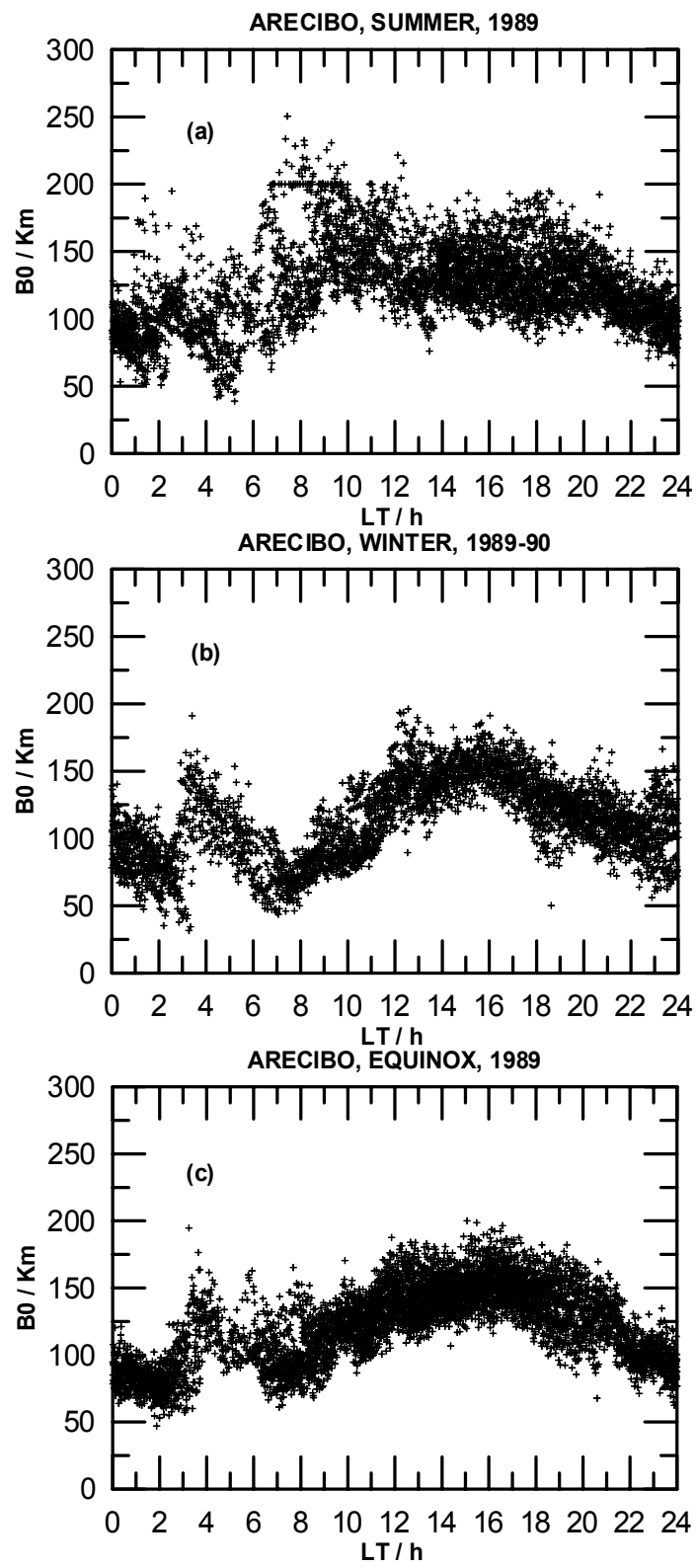

Fig. 2. A scatter plot showing diurnal variation of the parameter $B 0$ derived from the I.S. measurements at Arecibo for (a) summer, (b) winter and (c) equinox.

\section{Experimental data}

The electron density profiles used in the present study, as mentioned earlier, have been taken from the Arecibo observatory, Puerto Rico, USA. The effective height resolution is $0.6 \mathrm{~km}$, which was achieved by using s $4 \mu$ s pulse length. The time resolution is about $1 \mathrm{~min}$. The power profile is converted into an electron density profile by using a simultaneously measured electron to ion temperature ratio profile. The profile is finally calibrated by reading $N m \mathrm{~F} 2$ from an on-site ionosonde. We have employed the data for a period of solar maximum from March 1989 to January 1990, containing 

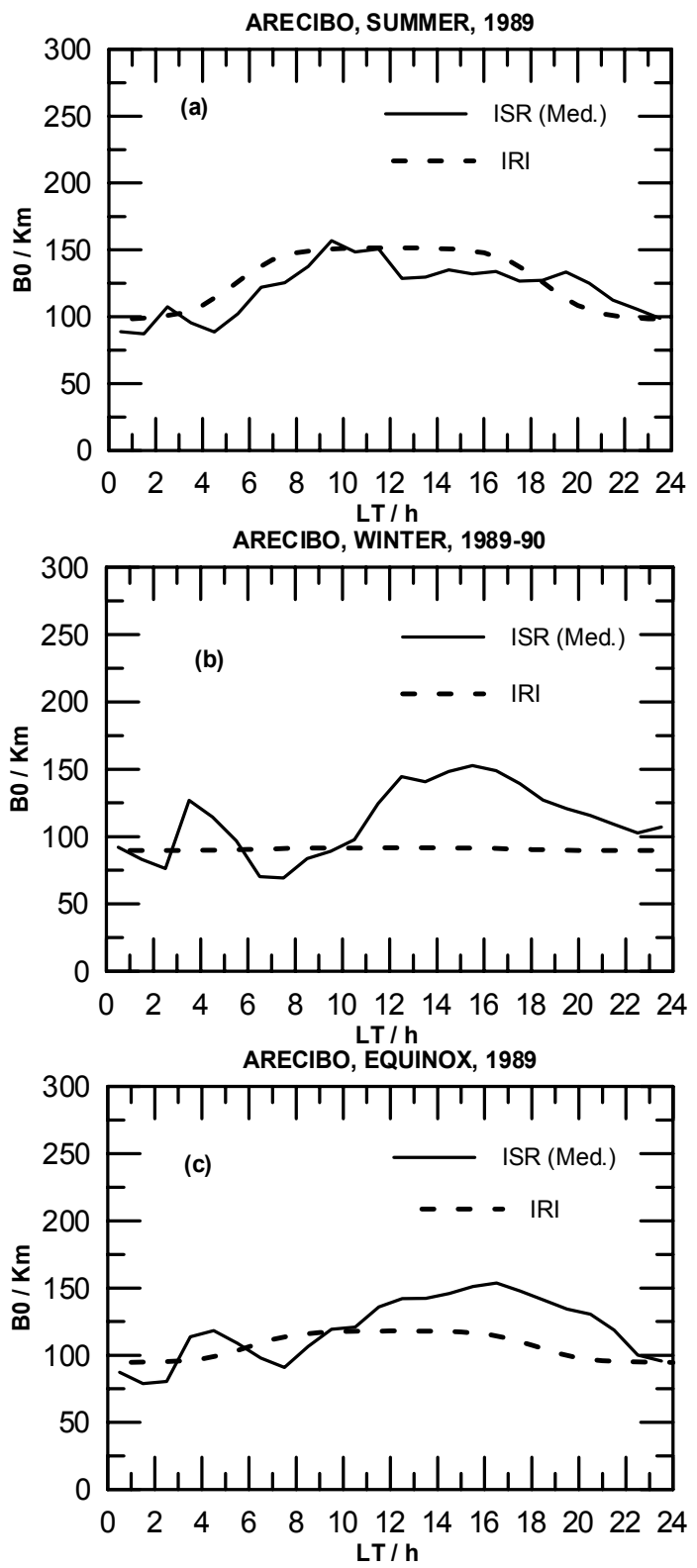

Fig. 3. Variation of median values of $B 0$ observed at Arecibo and those obtained from the IRI model for (a) summer, (b) winter and (c) equinox. The median values are shown as a solid line and the IRI model values are shown as a dashed line.

around $14171 \mathrm{Ne}$ profiles.

\section{Analysis and results}

The technique for obtaining $B 0, B 1$ from the observed profiles is demonstrated in Figs. 1a and b. These figures show some sample $\mathrm{Ne}$ profiles truncated at $0.24 \mathrm{Nm} \mathrm{F} 2$ below the F2 peak. The IRI profile function, described by Eq. (1), has been fitted to these profiles and the best $B 0, B 1$ parameters have been obtained. The best fit profiles are shown as solid lines and the IRI profiles are shown by dashed lines.

Since the IRI-2001 has $B 0, B 1$ models for the three seasons, summer, winter and equinox, we also have grouped the $N(e)$ profiles into these three seasons. For summer, we have 4675 profiles, for winter, 3709 profiles and for equinox, 5787 profiles. These profiles, as stated earlier, were truncated at $0.24 N m \mathrm{~F} 2$ and were then fitted to the IRI profile function, to find optimum $B 0, B 1$ parameters. Median values for each of these parameters were calculated for 30-min bins of local time. The $B 0, B 1$ values predicted by IRI-2001 (Bilitza, 2001) were obtained for RZ12 $=150$, day 15 of June, March and December, as representative of summer, equinox and winter, respectively.

Figures $2 \mathrm{a}-\mathrm{c}$ show mass plots of $B 0$ against local time for summer, winter and equinox seasons. It is observed that for the same local time and in the same season, $B 0$ shows a large scatter, and varies by as much as a factor of 2 during all the seasons. In Figs. 3a-c, we compare the plots of median $B 0$ obtained from the incoherent scatter measurements and the IRI model for summer, winter and equinox, respectively. It can be seen from Fig. 3a that during summer, the median value of $B 0$ varies from around $90 \mathrm{~km}$ during night to about $150 \mathrm{~km}$ in the pre-noon hours, and thereafter, it falls gradually to about $80 \mathrm{~km}$ around midnight. However, it shows a good agreement with the IRI predicted values. The percentage difference between the two is less than 15\%. During the winter and equinox seasons, as can be seen from Figs. $3 \mathrm{~b}$ and $\mathrm{c}$, the median value of $B 0$ increases from about $90 \mathrm{~km}$ in the morning (07:00 LT) to about $150 \mathrm{~km}$ in the afternoon (15:0016:00 LT) and thereafter, it falls to about $100 \mathrm{~km}$ around midnight. $B 0$ shows an increasing trend from 02:00 to 04:00 LT with values varying from $75 \mathrm{~km}$ to $175 \mathrm{~km}$ (during winter) and 80 to $120 \mathrm{~km}$ (during equinox). In this time period, IRI generally underestimates $B 0$. It may also be noted that the IRI predicted values of $B 0$ do not show any local time change during winter. The percentage difference between the IRI predicted and the median values varies from about 35 to $65 \%$ during winter and from about 15 to $35 \%$ during equinox, for the local times between 12:00 to 20:00 LT. The difference between the two, outside this time period, remains at less than $20 \%$ for both the seasons.

Diurnal variations of mass plots of the parameter $B 1$ are shown in Figs. 4a-c for summer, winter and equinox, respectively. It can be seen that $B 1$ shows a large dispersion during the night hours, with values varying between 1 and 6 . The median values and the IRI predicted values of $B 1$ are shown in Figs. 5a-c. The median values vary between 1.45 and 3, in general. The IRI values exhibit more or less a similar diurnal trend, but with somewhat higher values during all the seasons and at all local times.

The thickness and shape parameters are expected to show solar activity variations, as seen in IRI-2001 (Bilitza, 2001). We have, therefore, compared in Figs. 6a-d the median values of these parameters for the solar maximum (1989-1990) with those obtained earlier by us (Mahajan and Sethi, 2001) for solar minimum (1974-1977), along with the IRI predicted values. For low solar activity, the predicted $B 0, B 1$ (Bilitza, 2001) values were obtained for RZ12 $=15$, day 15 

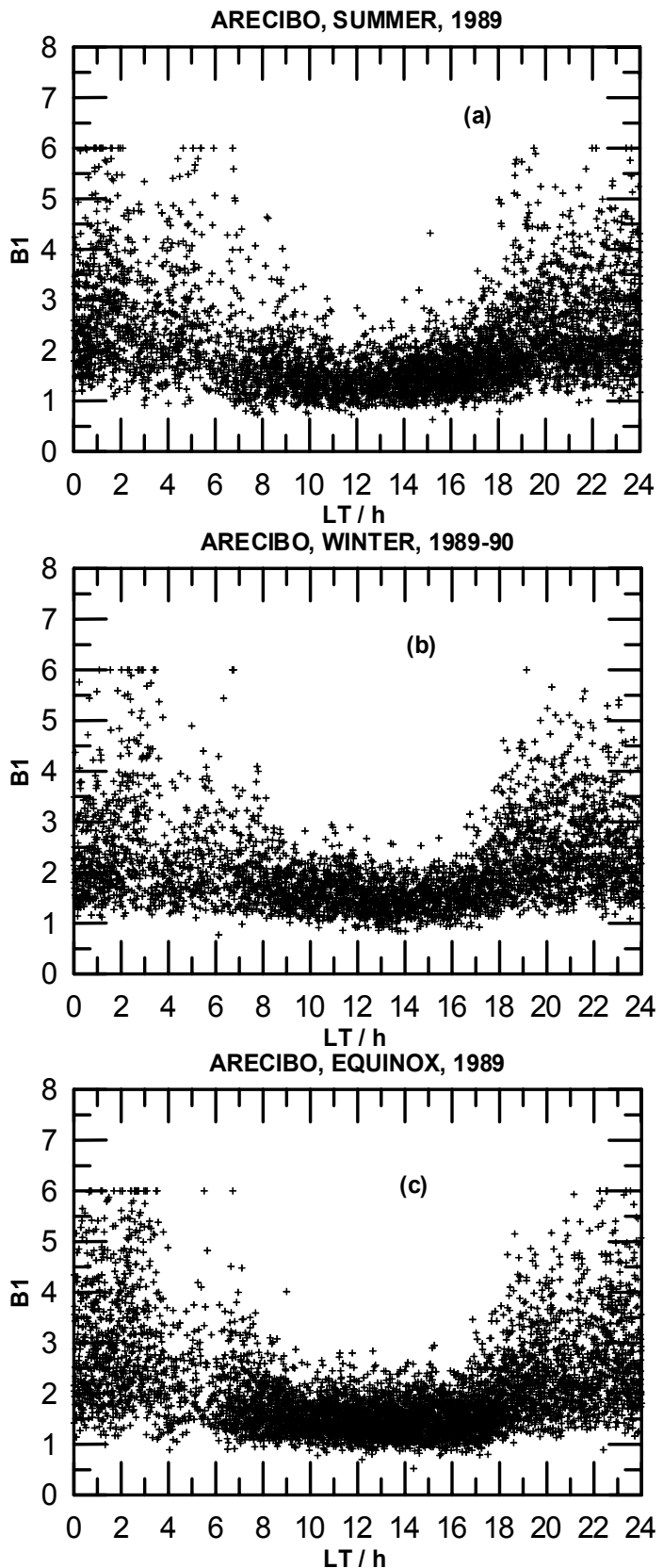

Fig. 4. A scatter plot showing diurnal variation of the parameter $B 1$ derived from the I.S. measurements at Arecibo for (a) summer, (b) winter and (c) equinox.

of June, March and December, as representative of summer, equinox and winter, respectively. The $B 0, B 1$ values thus obtained for low and high solar activity periods are averaged over three months to represent all seasons. It can be seen from Figs. $6 \mathrm{a}$ and $\mathrm{b}$ that the parameter $B 0$ increases by more than $50 \%$ from low solar activity to high solar activity, while the IRI predicted values show an increase by about $40 \%$ during daytime and by about $20 \%$ during the night. As seen from the Figs. $6 \mathrm{c}$ and d, the parameter $B 1$ decreases by about $20 \%$ from low solar activity to high solar activity. However, the
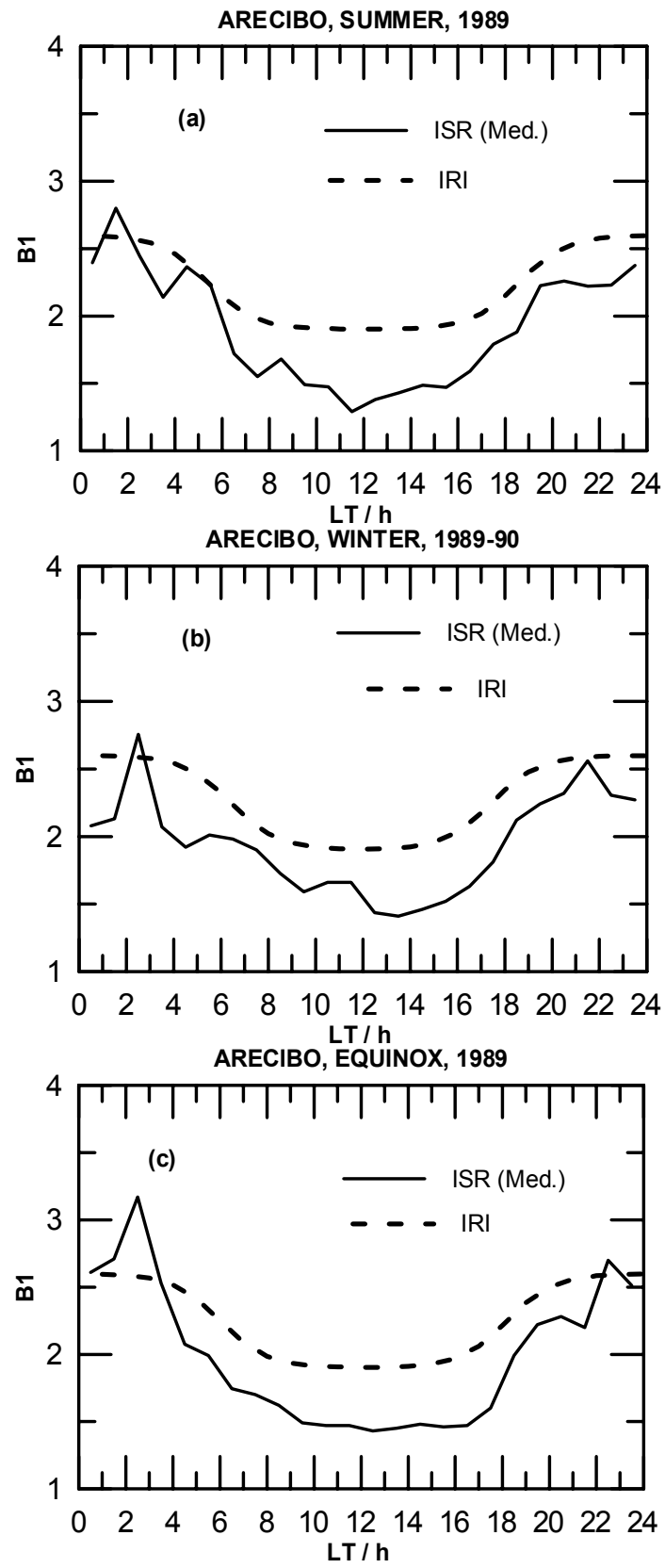

Fig. 5. Variation of median values of $B 1$ observed at Arecibo and those obtained from the IRI model for (a) summer, (b) winter and (c) equinox. The median values are shown as a solid line and the IRI model values are shown as a dashed line.

IRI does not exhibit any solar activity dependence.

\section{Conclusions}

Comparison of the IRI-2001 predicted $B 0$ parameter with those observed at Arecibo show a fair agreement during summer for most of the local times. A larger difference between the two occurs during winter and equinox seasons, particularly between 12:00 to 20:00 LT, when the IRI underesti- 

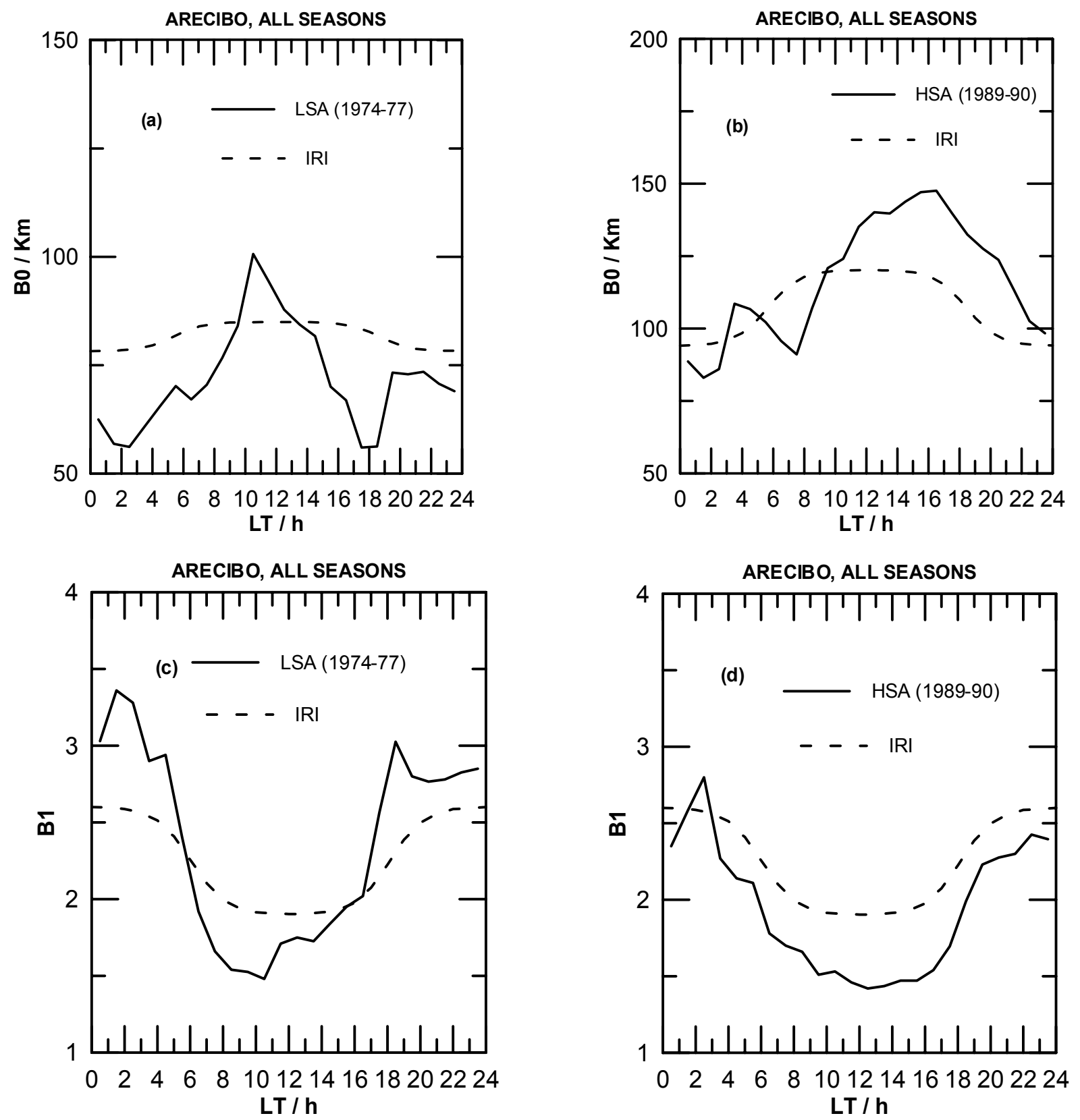

Fig. 6. Plots showing the median values of $B 0, B 1$ parameters obtained from the I.S. measurements, along with the IRI model during low and high solar activity periods. The median values are shown as solid lines and the IRI model values are shown as dashed lines. Low and high solar activity values of $B 0$ are shown in (a) and (b) and of $B 1$ in (c) and (d), respectively.

mates $B$. During winter, the IRI does not show any local time change for the parameter $B 0$. For other local times, the discrepancies are the least. For $B 1$, the model and the observations show a similar diurnal trend, with IRI showing somewhat higher values for all the seasons and for all local times. In contrast to our observations, IRI does not exhibit any solar activity dependence for $B 1$. The parameters $B 0$, $B 1$ obtained from measurements can be used as input to the IRI model, in order to achieve a more accurate shape of the electron density profile in the F2-region below the peak.
Acknowledgement. The Arecibo observatory is one of the major facility of the National Astronomy and Ionosphere Center, operated by Cornell University under a cooperative agreement with the $\mathrm{Na}$ tional Science Foundation. We are thankful to Qihou Zhou, Arecibo Observatory, HC3 Box 53995 Arecibo, PR 00612, Puerto Rico, USA for supplying us data. K. K. Mahajan is thankful to Council of Scientific and Industrial Research (CSIR) for the award of Emeritus Scientist position during the progress of this work.

Topical Editor M. Lester thanks two referees for their help in evaluating this paper. 


\section{References}

Aggarwal, S., Venkatachari, R., Sachdeva, V. P., and Jain, V. C.: Comparison of electron density profiles for Delhi with corresponding profiles obtained from IRI-90, Adv. Space Res., 18, 6, 39-40, 1996.

Adeniyi, J. O. and Radicella, S. M.: Variation of bottomside profile parameters $B 0$ and $B 1$ at high solar activity for an equatorial station, J. Atmos. Solar-Terr. Phys., 60, 1123-1127, 1998.

Bilitza, D. (Ed): The International Reference Ionosphere, NSSDC 90-2, World Data Center A, Rockets and Satellites, Greenbelt, USA, 1990.

Bilitza, D.: F1 and bottomside region in IRI - Status report, Proc. Of the IRI task force Activity 1995, IC/IR/96/14, (Ed) Radicella, S. M., Trieste, May 1996.

Bilitza, D., Radicella, S. M., Reinisch, B. W., Adeniyi, J. O., Mosert, M. E., Gonzalez, S., Zhang, S. R., and Obrou, O.: New $B 0$ and $B 1$ Models for IRI, Adv. Space Res., 25, 1, 89-95, 2000.

Bilitza, D.: International Reference Ionosphere 2000, Radio Science, 36, 2, 261-275, 2001.

De Gonzalez, M. M.: Observed and model $N(h)$ profiles for two Argentine stations, Adv. Space Res., 18, 6, 53-56, 1996.

Gulyaeva, T. L.: Progress in ionospheric informatic based on electron density profile analysis of ionograms, Adv. Space Res., 7,
2, 51-60, 1987.

Gulyaeva, T. L., Mahajan, K. K., and Sethi, N. K.: Modification of IRI half-density height option for low latitudes, Adv. Space Res., 18, 6, 149-152, 1996.

Huang, X. and Reinisch, B. W.: Fitting the IRI profile function to measured profiles, IRI Task Force Activity 1996, International Center of Theoretical Physics, Trieste, Italy, 1996.

Mahajan, K. K., Kohli, R., Sethi, N. K., and Pandey, V. K.: Variability of the F-region parameter $h 0.5$, Adv. Space Res., 15, 2, 51-60, 1995.

Mahajan, K. K. and Sethi, N. K.: Empirical models of parameters B0, $B 1$ from Arecibo radar measurements, Adv. Space Res., 27, 1, 17-20, 2001.

Pandey, V. K. and Sethi, N. K.: Comparison of $h m F 2$ and midday bottomside electron density profile obtained from IRI and incoherent scatter measurements, Adv. Space Res., 18, 6, 33-37, 1996.

Ramakrishnan, S. and Rawer, K.: Model electron density profiles obtained by empirical procedures, Space Research XII, 1253, Akademie-Verlag, Berlin, German Democratic Republic, 1972.

Sethi, N. K., Mahajan, K. K. and Pandey, V. K.: Bottomside parameters $B 0, B 1$ from Arecibo incoherent scatter radar measurements, Adv. Space Res., 25, 1, 97-100, 2000. 